\section{KÖPFE DER SOZIALWIRTSCHAFT: CHRISTIAN TÖLKEN}

\section{»Freude am Gelingen «}

SOZIALwirtschaft befragt, in Anlehnung an Marcel Prousts Fragebogen, Führungskräfte der Sozialwirtschaft nach ihren Erfahrungen und Meinungen, nach ihrem Arbeiten und Leben. In diesem Heft antwortet Christian Tölken von den Rummelsberger Anstalten.

SOZIALwirtschaft: Was war Ihre Motivation, Führungskraft in der Sozialwirtschaft zu werden?

Christian Tölken: An einer guten Sache beteiligt zu sein.

SOZIALwirtschaft: Was ist für Sie Erfolg in der Sozialwirtschaft?

Christian Tölken: Wenn es wieder einmal gelungen ist, die Lage eines in Nöten befindlichen Menschen oder einer Menschengruppe wirksam und nachhaltig zu verbessern oder zumindest zu erleichtern.

SOZIALwirtschaft: Welches Ereignis in Ihrem Leben hat Ihre berufliche Laufbahn am meisten beeinflusst?

Christian Tölken: Die Geburt unserer Drillinge.

SOZIALwirtschaft: Welche Rolle spielten Zufall und Glück in Ihrer Karriere?

Christian Tölken: Eine große.

SOZIALwirtschaft: Haben Sie Vorbilder?

Christian Tölken: Alfred Herrhausen, Dietrich Bonhoeffer, Anne Frank.

SOZIALwirtschaft: Welche Ihrer Eigenschaften war beruflich besonders wichtig?

Christian Tölken: Zuhören, beurteilen und rasch entscheiden zu können.

SOZIALwirtschaft: Welche waren eher hinderlich?

Christian Tölken: Zu schnell zu vertrauen und zu lange daran festzuhalten.

SOZIALwirtschaft: Was kennzeichnet Ihren Arbeitsstil?

Christian Tölken: Auf das Wesentliche konzentrieren und das Richtige tun.

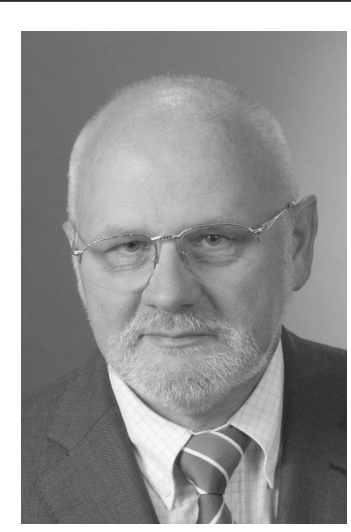

Christian Tölken, 1946 in Bad Oeynhausen geboren, ist seit 1981 Mitarbeiter der Rummelsberger Anstalten. In den ersten beiden Jahren war er Assistent des Vorstandes, dann von 1982 bis 1989 Leiter des Wichernhauses Altdorf. Von 1990 bis 2004 war er Mitglied der Anstaltsleitung, zunächst als Personalabteilungsleiter, von 1994 bis 2004 war er als Verwaltungsdirektor das Bevollmächtigte Vorstandsmitglied und gleichzeitig Leiter der Hauptverwaltung und der Zentralabteilung Finanzwirtschaft und Grundsatzfragen. Ehrenamtlich war er u. a. Mitglied des Beirates der Ecclesia-Versicherungsgruppe und des Zentralbeirates der Bank für Sozialwirtschaft. Nach der großen Strukturreform der Rummelsberger Anstalten wurde Christian Tölken am 12. Mai 2005 zum Hauptgeschäftsführer der unternehmensführenden Muttergesellschaft »Die Rummelsberger Dienste für Menschen gGmbH « bestellt. Im Rahmen des Rummelsberger Unternehmensentwicklungsprozesses war er zudem vom 12. Mai bis 31. Dezember 2004 Gründungsgeschäftsführer aller weiteren gemeinnützigen Rummelsberger Tochtergesellschaften. E-Mail toelken.christian@rummelsberger.net

SOZIALwirtschaft: Was war Ihr bisher größter beruflicher Erfolg?

Christian Tölken: Die vollständig gelungene Strukturreform eines großen Sozialunternehmens.

SOZIALwirtschaft: Ihr größter Misserfolg?

Christian Tölken: Ein politisches Mandat anzustreben.

SOZIALwirtschaft: Welche Eigenschaften schätzen Sie besonders an Ihren Mitarbeitern?

Christian Tölken: Zuverlässigkeit, Eigenständigkeit, Wirksamkeit, Freude am Gelingen.

SOZIALwirtschaft: Welches Verhalten von Mitarbeitern macht Ihnen am meisten Probleme?

Christian Tölken: Angst und Unaufrichtigkeit.

SOZIALwirtschaft: Welche Eigenschaften sind bei Führungskräften in der Sozialwirtschaft besonders wertvoll, welche besonders hinderlich?

Christian Tölken: Besonders wertvoll ist es, für Ziele sorgen und sie verwirklichen zu können und besonders hinderlich ist es, den Menschen nichts zuzutrauen und negativ zu denken. 
SOZIALwirtschaft: Haben Sie ein persönliches Motto für Ihr eigenes Führungskonzept?

Christian Tölken: Mehr sein als scheinen, Stärken nutzen, Menschen fördern, vorausdenken und die Umgebung mitkommen lassen.

SOZIALwirtschaft: Welche strukturellen Probleme in der Sozialwirtschaft erschweren am meisten unternehmerische Erfolge?

Christian Tölken: Vergütungsstruktur, überholte Hierarchien und Vereinsstrukturen, mangelnde Corporate Governance, Ausmaß an Regulierung.

SOZIALwirtschaft: Wie sehen Sie die Chancen und Perspektiven für Betriebe und Unternehmen in der Sozialwirtschaft?

Christian Tölken: Chancen und Perspektiven sind langfristig betrachtet in den wichtigsten Handlungsfeldern (Gesundheitswesen, Pflege, Behindertenhilfe) teilweise ausgezeichnet - bei rascher Anpassung an wandelnde Rahmenbedingungen.

\section{SOZIALwirtschaft: Wie entspannen Sie sich?}

Christian Tölken: Lesen, wandern, Musik hören, schreiben, fotografieren und Bildbearbeitung.

SOZIALwirtschaft: Was werden Sie machen, wenn Sie nicht mehr Führungskraft sind?

Christian Tölken: Vielleicht ein Unternehmen gründen. Ganz sicher aber ehrenamtlich arbeiten und hin und wieder den Fischern im Hafen von Paphos (Zypern) zuzuschauen bei einem Glas guten Weines, einem guten Käse und einem Fladen Brot.

\section{Köpfe der Sozialwirtschaft}

Den Fragebogen von SOZIALwirtschaft beantworteten bisher:

Dr. Gitta Trauernicht, Ministerin für Soziales, Gesundheit, Familie, Jugend und Senioren in Schleswig-Holstein

SOZIALwirtschaft 1/2005

Günther Famulla, Vorsitzender des Vorstandes des Paritätischen Wohlfahrtsverbandes, Landesverband Niedersachsen e. V.

SOZIALwirtschaft 2/2005

Franz J. Stoffer, Geschäftsführer der CBT Caritas-Betriebsführungs- und Trägergesellschaft mbH, Köln SOZIALwirtschaft 3/2005

Gabriele Sörgel, Sprecherin des Vorstandes der Stadtmission Nürnberg e. V.

SOZIALwirtschaft 4/2005

Rainer Brückers, Bundesgeschäftsführer der Arbeiterwohlfahrt Bundesverband e. V. SOZIALwirtschaft 5/2005

Josef Seekircher, Leiter der Sozial- und Jugendbehörde der Stadt Karlsruhe SOZIALwirtschaft 1/2006

Thomas Dane, Mitglied des Vorstands des Diakonischen Werkes Berlin-Brandenburg-schlesische Oberlausitz

SOZIALwirtschaft 2/2006

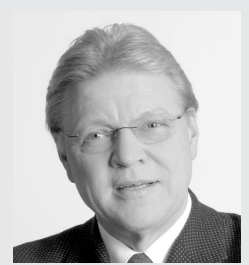

Klaus-Dieter Kottnik, Vorstandsvorsitzender der Diakonie Stetten SOZIALwirtschaft 3/2006

Das Jahresabonnement der Zeitschrift SOZIALwirtschaft kostet 87,- Euro. Der Kombi-Preis für die Zeitschrift SOZIALwirtschaft und den Informationsdienst SOZIALwirtschaft aktuell beträgt 149,jährlich. Der Preis für ein Einzelheft von SOZIALwirtschaft beläuft sich auf 19,- Euro. Alle Preise zuzüglich Porto- und Versandkosten.

Nomos Verlagsgesellschaft, 76520 Baden-Baden, Telefon 07221 2104-39, Fax 07221210443 , E-Mail hohmann@nomos.de 